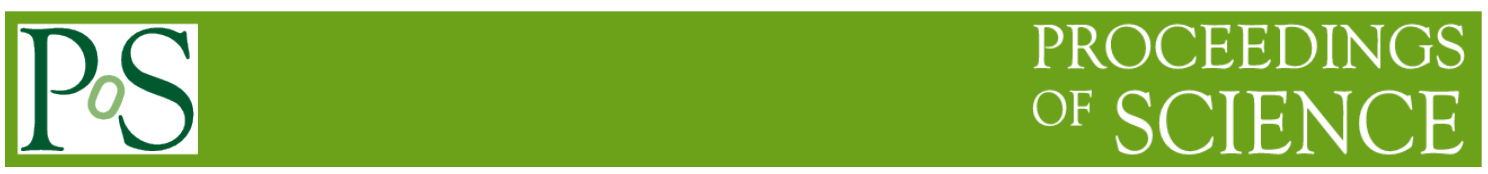

\title{
Precision measurement of the antiproton-to-proton ratio by the Alpha Magnetic Spectrometer on the International Space Station
}

\author{
Andrew lan Chen ${ }^{*}$ on behalf of the AMS Collaboration \\ Massachusetts Institute of Technology \\ E-mail: achen7@mit.edu \\ A precision measurement, with the Alpha Magnetic Spectrometer, of the antiproton-to-proton \\ ratio in the primary cosmic rays is presented. The measurement is made in the rigidity range \\ from 1 to $450 \mathrm{GV}$ and is based on 300,000 antiproton events. The measurement increases the \\ precision and significantly extends the high rigidity range beyond previous observations.
}

38th International Conference on High Energy Physics

3-10 August 2016

Chicago, USA

* Speaker 


\section{Introduction}

We report the measurement of the antiproton flux, antiproton-to-proton flux ratio, and properties of elementary particle fluxes in primary cosmic rays (CR) by the Alpha Magnetic Spectrometer (AMS). This measurement published in [1] of the antiproton flux, and of the antiproton-to-proton flux ratio in CR covers the rigidity range of 1 to $450 \mathrm{GV}$ and is based on $3.49 \times 10^{5}$ antiproton events and $2.42 \times 10^{9}$ proton events. The events were collected by AMS aboard the International Space Station (ISS) from May 19, 2011 to May 26, 2015.

The measurement of CR antiprotons $(\overline{\mathrm{p}})$ is complementary to the measurement of $\mathrm{CR}$ positrons $\left(\mathrm{e}^{+}\right)$presented in $[2,3]$. AMS has now measured the properties of all the elementary particles in the cosmos [1,3-5]. This allows for new observations of their properties. The data on $\mathrm{e}^{ \pm}$have already generated many interesting theoretical models. These models will be constrained by further measurements by AMS such as those described in [6].

\section{The AMS Detector and Event Selection}

To measure the $\overline{\mathrm{p}}$ flux to $1 \%$ accuracy requires a separation power between $\overline{\mathrm{p}}$ and $\mathrm{p}$ of $\sim 10^{6}$. We use all the AMS detector elements [7] for particle identification and selection among the 65 billion CR triggers recorded. The detector elements are: the silicon tracker surrounded by permanent magnet, the time of flight counters (TOF), the anticoincidence counters (ACC), the transition radiation detector (TRD), the ring imaging Čerenkov detector (RICH), and the electromagnetic calorimeter (ECAL).

The nine layer tracker is used to measure the rigidity $\mathrm{R}$ (momentum per unit of charge) of $\mathrm{CR}$ and to differentiate between positive and negative particles. For particles with absolute charge $|\mathrm{Z}|=1$ the maximum detectable rigidity is $2 \mathrm{TV}$. CR events are selected with $\chi^{2} /$ d.o.f. of the track fit less than 10 . Only primary $\mathrm{CR}$ are selected by requiring $\mathrm{R}$ to be greater than 1.2 times the geomagnetic cutoff.

The TOF measures charge, selects downward-going particles, and measures velocity with a resolution of $\Delta \beta / \beta=4 \%$. The ACC is used to reject CR entering from the side. The TRD separates $\overline{\mathrm{p}}$ and $\mathrm{p}$ from $\mathrm{e}^{-}$and $\mathrm{e}^{+}$using the $\Lambda_{\text {TRD }}$ estimator constructed from the ratio of the loglikelihood probability of the $e^{ \pm}$hypothesis to that of the $\bar{p}$ or p hypothesis [3]. The $d E / d x$ measured in the TRD, the TOF, and the tracker are required to be consistent with $|\mathrm{Z}|=1$.

For $|\mathrm{Z}|=1$ the RICH has a velocity resolution $\Delta \beta / \beta=0.1 \%$ which ensures separation of $\bar{p}$ and $\mathrm{p}$ from light particles $\left(\mathrm{e}^{ \pm}\right.$and $\left.\pi^{ \pm}\right)$. The separation using the RICH velocity measurement and TRD estimator is show in Figure 1. Light negative mesons are present only at rigidities below $10 \mathrm{GV}$. Finally, the ECAL is used to separate $\overline{\mathrm{p}}$ and $\mathrm{p}$ from $\mathrm{e}^{-}$and $\mathrm{e}^{+}$based on shower shape. The ECAL is also used for selecting a pure sample of $\mathrm{e}^{-}$.

\section{Data Analysis}

The combination of information from the detector elements enables the efficient separation of the $\bar{p}$ signal from background. This is done using template fitting. The number of observed $\bar{p}$ signal events and its statistical error in each bin is found by fitting signal and background templates to data. The signal template is defined using the high-statistics proton data sample. This is possible because the distribution of the variables for the template definition is the same 
for $\bar{p}$ and $p$ if they are both reconstructed with a correct charge-sign. This is done for a detailed Monte Carlo simulation based on the GEANT 4.10.1 package [8]. It has also been verified using $\overline{\mathrm{p}}$ and $\mathrm{p}$ data for $2.97 \leq|\mathrm{R}|<18.0 \mathrm{GV}$.

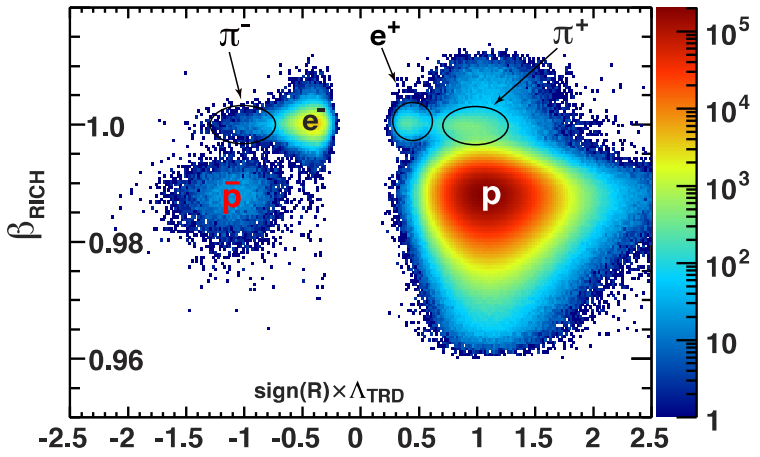

Figure 1: Data samples for the absolute rigidity range 5.4-6.5 GV clearly seperated by the RICH and TRD.

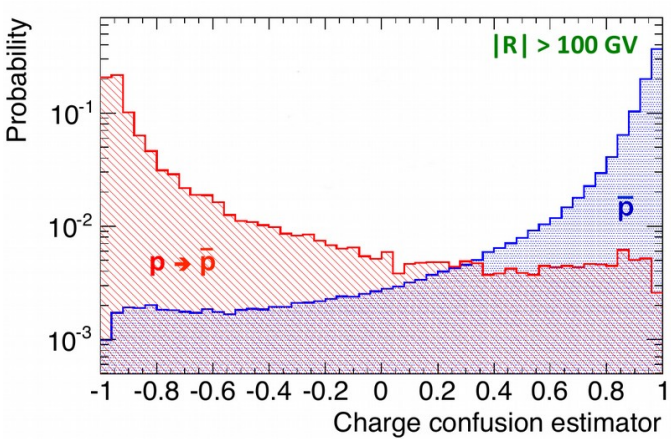

Figure 2: The performance of the charge confusion estimator.

For rigidities above $10 \mathrm{GV}$, separating $\overline{\mathrm{p}}$ from charge confusion $\mathrm{p}$ becomes the primary challenge. These are $p$ which are reconstructed with negative rigidity due to the finite tracker resolution. This separation is done using a charge confusion estimator, $\Lambda_{\mathrm{CC}}$, which is defined using the boosted decision tree technique. The separation power of $\Lambda_{\mathrm{CC}}$ is shown in Figure 2.

The template fit is then done in the two-dimensional, $\Lambda_{\mathrm{TRD}}-\Lambda_{\mathrm{CC}}$, plane. To fit the data three template shapes are defined: $\overline{\mathrm{p}}$ with correctly reconstructed charge sign, $\mathrm{e}^{-}$, and charge confusion $\mathrm{p}$. The $\mathrm{p}$ template is based on the Monte Carlo simulation and verified with $\mathrm{p}$ test beam data. An example of the fit for the rigidity bin $175-211 \mathrm{GV}$ is shown in Figure 3. All together there are total of $3.49 \times 10^{5} \overline{\mathrm{p}}$ events in the data.
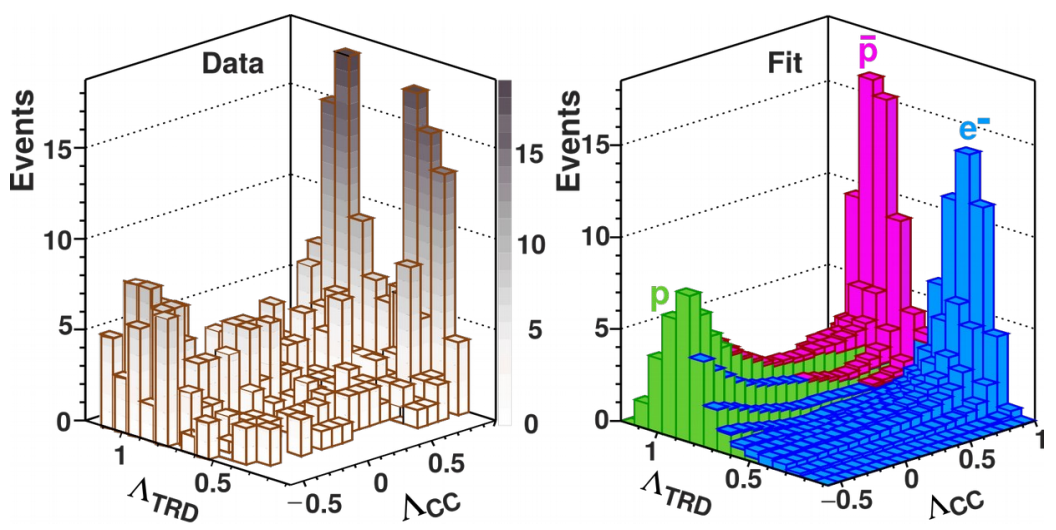

Figure 3: On the left, negative reconstructed rigidity data in the 175-211 GV absolute rigidity bin. On the right, the fit using the $\bar{p}$ signal template, the $e^{-}$background template, and the charge confusion $p$ background template. The $\chi^{2}$ of the fit is 138 for 154 degrees of freedom.

Corrected for bin-to-bin migration using an iterative unfolding techinique, we have the number of $\overline{\mathrm{p}}$ events at the top of the AMS, $N_{i}^{\bar{p}}$. This is for a given absolute rigdity bin, $R_{i}$. We can then compute the isotropic flux in the bin $i$ with width $\Delta R_{i}$ as 


$$
\Phi_{i}^{\bar{p}}\left(R_{i}\right)=\frac{N_{i}^{\bar{p}}}{A_{i}^{\bar{p}} T_{i} \Delta R_{i}}
$$

where $A_{i}^{\bar{p}}$ is the corresponding effective acceptance including geometric and efficiency effects, and $T_{i}$ is the exposure time.

\section{Systematic Errors}

The first source of errors on the $\overline{\mathrm{p}}$ flux and $(\overline{\mathrm{p}} / \mathrm{p})$ flux ratio are the effects on $N_{i}^{\bar{p}}$. Variation of the geomagnetic cutoff factor in the range 1.2 to 1.4 shows a systematic uncertainty of $\sim 1 \%$ at $1 \mathrm{GV}$ and negligible above $2 \mathrm{GV}$. The cutoff is calculated by backtracing [5] using the most recent IGRF geomagnetic model [9]. The analysis is also repeated in each rigidity bin with different sets of selections. This resulting uncertainty from event selection amounts to $4 \%$ at $1 \mathrm{GV}$, falls to $0.5 \%$ at $10 \mathrm{GV}$, and rises to a maximum of $6 \%$ at $450 \mathrm{GV}$.

Uncertainties of the proton flux in the TV region are estimated by varying the spectral index of the proton flux within the accuracy of the AMS proton measurement [5]. The systematic errors due to the the shape of the charge confusion proton template originate from uncertainties of the proton rigidity resolution function. They are estimated by comparing the charge confusion predicted by the Monte Carlo simulation with the charge confusion obtained from the fit. The systematic error from these effects are estimated to be to $<1 \%$ below $30 \mathrm{GV}$ rising to $12 \%$ at $450 \mathrm{GV}$.

The systematic errors on the folded acceptances, $A_{i}^{\bar{p}}$ and $A_{i}^{p}$, are attributed to the the uncertainties in the interaction cross sections for protons and antiprotons in the detector materials. This is estimated by varying the $\overline{\mathrm{p}}$ and $\mathrm{p}$ interaction cross sections in the Monte Carlo simulation. The corresponding error on $A_{i}^{\bar{p}}$ is found to be $4 \%$ at $1 \mathrm{GV}$ and $\sim 1 \%$ above 50 GV. The error on $A_{i}^{p}$ is found to be $2.5 \%$ at $1 \mathrm{GV}$ and $\sim 1 \%$ above $50 \mathrm{GV}$.

The systematic errors from correcting for bin-to-bin migration are $1 \%$ below $200 \mathrm{GV}$ and $1.5 \%$ at $450 \mathrm{GV}$. These systematic errors partially cancel in the $(\overline{\mathrm{p}} / \mathrm{p})$ flux ratio, yielding uncertainties of $1 \%$ at $1 \mathrm{GV}$ and $<0.5 \%$ above $2 \mathrm{GV}$.

The error on the absolute rigidity scale was estimated by comparing the $\mathrm{e}^{ \pm}$energies measured in the ECAL with the momentum measured in the tracker. The corresponding errors on the $\bar{p}$ and $p$ fluxes are negligble below $10 \mathrm{GV}$ and gradually increase to $\sim 1 \%$ at $450 \mathrm{GV}$.

The uncertainty from the Monte Carlo simulation based template shapes are also nonnegligible for $|\mathrm{R}|>30 \mathrm{GV}$. As reported in [1] and to be detailed seperately, these uncertanties

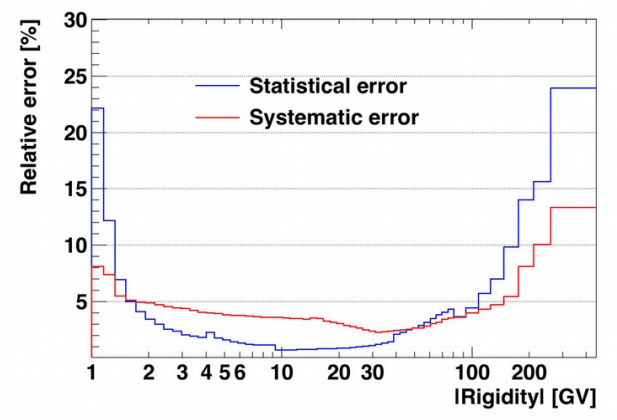

Figure 4: The error breakdown for the $\bar{p} / p)$ flux ratio. 
were verified by a completely independent data driven analysis. This was one of several independent analyses that were performed. The results of these independent analyses are consistent.

The independent sources of systematic error are added together in quadrature to arrive at the total systematic error. The errors on the $(\overline{\mathrm{p}} / \mathrm{p})$ flux ratio are shown in Figure 4. At high and low R statistical error dominates. With continued data taking possible through the end of ISS service, AMS will continue to improve this measurement.

\section{Results}

The measured $(\overline{\mathrm{p}} / \mathrm{p})$ flux ratio is presented in Figure 5. A table is available in [1].

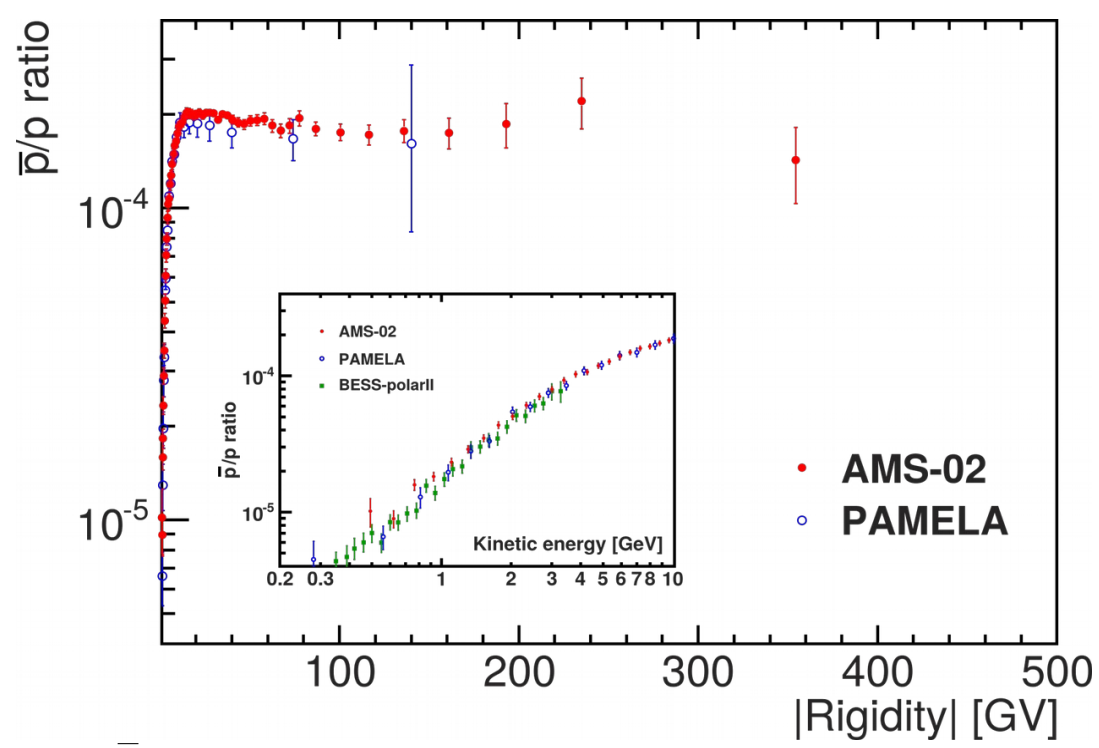

Figure 5: The $\bar{p} / p$ flux ratio as measured by AMS along with previous measurments by BESS [11] and PAMELA [10].

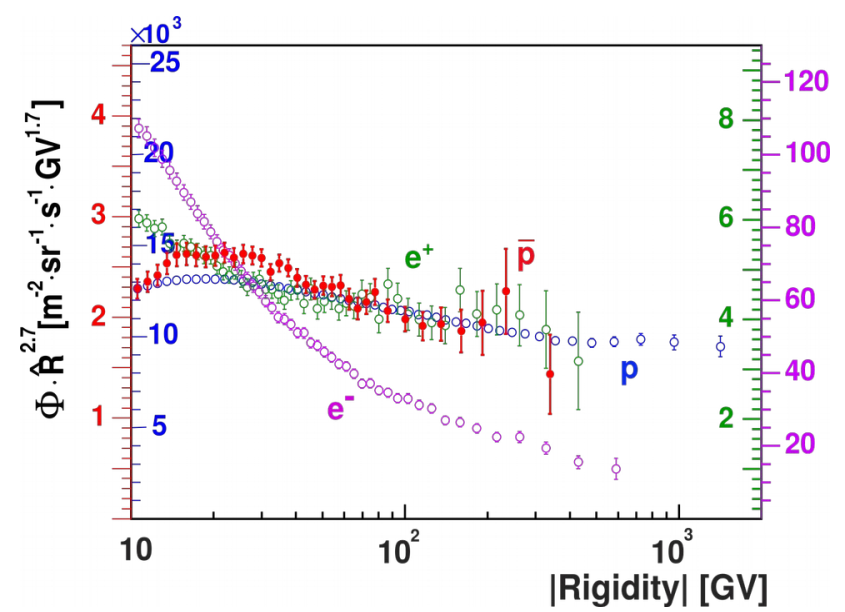

Figure 6: The elementary particle fluxes in primary cosmic rays as measured by AMS.

Compared with earlier experiments $[10,11]$, the AMS results extend the rigidity range to 450 $\mathrm{GV}$ and increase precision. A new observation is that the ratio appears to be rigidity independent at some point. To estimate the lowest rigidity above which the $(\overline{\mathrm{p}} / \mathrm{p})$ flux ratio is rigidity independent, we use intervals with varying starting rigidities above $10 \mathrm{GV}$ and ending at the 
final bin $450 \mathrm{GV}$. Each interval is split into two sections. Each of the sections is fit with a constant. The lowest starting rigidity that gives consistent mean values at $90 \%$ C.L. defines the lowest rigidity above which the $(\overline{\mathrm{p}} / \mathrm{p})$ flux ratio is rigidity independent. This yields $60.3 \mathrm{GV}$.

AMS has now simultaneously measured the fluxes of $\mathrm{e}^{ \pm}[4]$ and of $\mathrm{p}$ [5] with a single detector. This enables us to study the overall rigidity dependent behavior of different fluxes as shown in Figure 6 . The fluxes for $\bar{p}, \mathrm{p}$, and $\mathrm{e}^{+}$show similar rigidity dependence above $\sim 60 \mathrm{GV}$, whereas the rigidity dependence of the $\mathrm{e}^{-}$flux shows different behavior. Correspondingly, in the absolute rigidity range below $60 \mathrm{GV}$, the $(\overline{\mathrm{p}} / \mathrm{p}),\left(\overline{\mathrm{p}} / \mathrm{e}^{+}\right)$, and $\left(\mathrm{p} / \mathrm{e}^{+}\right)$flux ratios each reach a maximum. In the absolute rigidity range $\sim 60$ to $\sim 500 \mathrm{GV}$, the $(\overline{\mathrm{p}} / \mathrm{p}),\left(\overline{\mathrm{p}} / \mathrm{e}^{+}\right)$, and $\left(\mathrm{p} / \mathrm{e}^{+}\right)$flux ratios show no rigidity dependence. These are new observations of the properties of elementary particles in the cosmos.

\section{References}

[1] M. Aguilar et al., Antiproton Flux, Antiproton-to-Proton Flux Ratio, and Properties of Elementary Particle Fluxes in Primary Cosmic Rays Measured with the Alpha Magnetic Spectrometer on the International Space Station, Phys. Rev. Lett. 117, 091103 (2016).

[2] V. Vagelli, Precision Measurement of the $\left(e^{+}+e^{-}\right)$Flux in Primary Cosmic Rays from $0.5 \mathrm{GeV}$ to 1 $\mathrm{TeV}$ with the Alpha Magnetic Spectrometer on the International Space Station, in proceedings of the 38th International Conference on High Energy Physics PoS (ICHEP2016) 070.

[3] M. Aguilar et al., First Result from the Alpha Magnetic Spectrometer on the International Space Station: Precision Measurement of the Positron Fraction in Primary Cosmic Rays of 0.5-350 GeV, Phys. Rev. Lett. 110, 141102 (2013); L. Accardo et al., High Statistics Measurement of the Positron Fraction in Primary Cosmic Rays of 0.5-500 GeV with the Alpha Magnetic Spectrometer on the International Space Station, Phys. Rev. Lett. 113, 121101 (2014).

[4] M. Aguilar et al., Electron and Positron Fluxes in Primary Cosmic Rays Measured with the Alpha Magnetic Spectrometer on the International Space Station, Phys. Rev. Lett. 113, 121102 (2014).

[5] M. Aguilar et al., Precision Measurement of the Proton Flux in Primary Cosmic Rays from Rigidity 1 GV to 1.8 TV with the Alpha Magnetic Spectrometer on the International Space Station, Phys. Rev. Lett. 114, 171103 (2015).

[6] A. Oliva, Precision Measurement of Boron-to-Carbon and Carbon-to-Helium flux ratio in Cosmic Rays from 2 GV to 2 TV with the Alpha Magnetic Spectrometer on the International Space Station, in proceedings of the 38th International Conference on High Energy Physics PoS (ICHEP2016) 072 .

[7] A. Kounine, The Alpha Magnetic Spectrometer on the International Space Station, Int. J. Mod. Phys. E 21, 1230005 (2012)

[8] S. Agostinelli et al., GEANT4: A Simulation toolkit, Nucl. Instrum. Meth. A506(2003) 250-303.

[9] E. Thébault et al., International Geomagnetic Reference Field: the 12th generation, Earth Planets Space 67,79(2015).

[10] O. Adriani et al., Measurement of the flux of primary cosmic ray antiprotons with energies of 60 MeV to $350 \mathrm{GeV}$ in the PAMELA experiment, JETP Lett. 96, 621 (2013).

[11] K. Yoshimura et al., Measurement of the Cosmic-Ray Antiproton Spectrum at Solar Minimum with a Long-Duration Balloon Flight over Antarctica, Phys. Rev. Lett. 108, 051102 (2012). 